\title{
REALISATION OF MICRO-SPECKLE PATTERNS FOR DIGITAL IMAGE CORRELATION ON DENTAL IMPLANTS
}

\author{
C. Cluydts ${ }^{1}$ and W. De Waele ${ }^{2}$ \\ ${ }^{1}$ Ghent University, Belgium \\ ${ }^{2}$ Ghent University, Laboratory Soete, Belgium
}

\begin{abstract}
In vitro experiments and finite element analyses can be used to investigate the mechanical performance of dental implant structures. But before experimental and mechanical evaluations can be carried out, the right material properties, test methods and measurement techniques should be pointed out. The material properties of dental implants and characteristics of the jaw bone are widely documented. Concerning the right experimental setup and measurement techniques no conclusive description is given. Digital Image Correlation (DIC) is currently a widespread method. It uses random speckle patterns that are correlated to accurately measure displacement and strains of 3D-structures. If accurate results are pursued, first a thorough optimisation of this measuring technique must be accomplished. The choices are made based on the expected effect of load application on a dental implant. In literature the expected displacements are quite small, magnitude $100 \mu \mathrm{m}$, which requires high accuracy measurements. The possibilities of using DIC on micro-scale for titanium components is discussed in this paper.
\end{abstract}

Keywords: Dental implants; Microscale; Digital Image Correlation; Speckle pattern.

\section{INTRODUCTION}

Dental implants are small, sturdy, titanium posts that look as natural replacements for missing teeth. The implants act as natural tooth roots; they can hold a crown or can be used to anchor dentures or bridge structures for partially or fully edentulous patients. Placement of the prosthesis is done in two steps. First the implant is surgically inserted in the maxilla (upper jaw bone) or mandible (lower jaw bone). In the second step placement of the abutment takes place when osseo-integration of the dental implant has occurred (bone has sufficiently grown back around the implant).

In several studies all-on-four implants for fully edentulous patients are evaluated. This is a complete dental arch supported by four implants; two straight implants in the anterior and two angled implants in the posterior. These last two implants have to be angulated because of the presence of alveolar nerves in the mandible, one at each side of the jaw bone, that have to be avoided. In patients who have been edentulous for a long time, the residual mandibular bone height above the mandibular nerves is insufficient. Consequently the angulation of the implant has to be sufficient to lead the implant past the nerve. Moreover the imposed angulation has some advantages; (i) an angulated implant makes it possible to anchor the implant in better quality anterior bone and offers improved support of the prosthesis; (ii) longer implants can be used in minimum bone volume, thereby increasing bone-to-implant contact. Neither do tilted implants have a negative effect on load distribution; on the contrary good results and survival rates between 92 and 100\% have been accomplished [1, 2]. 


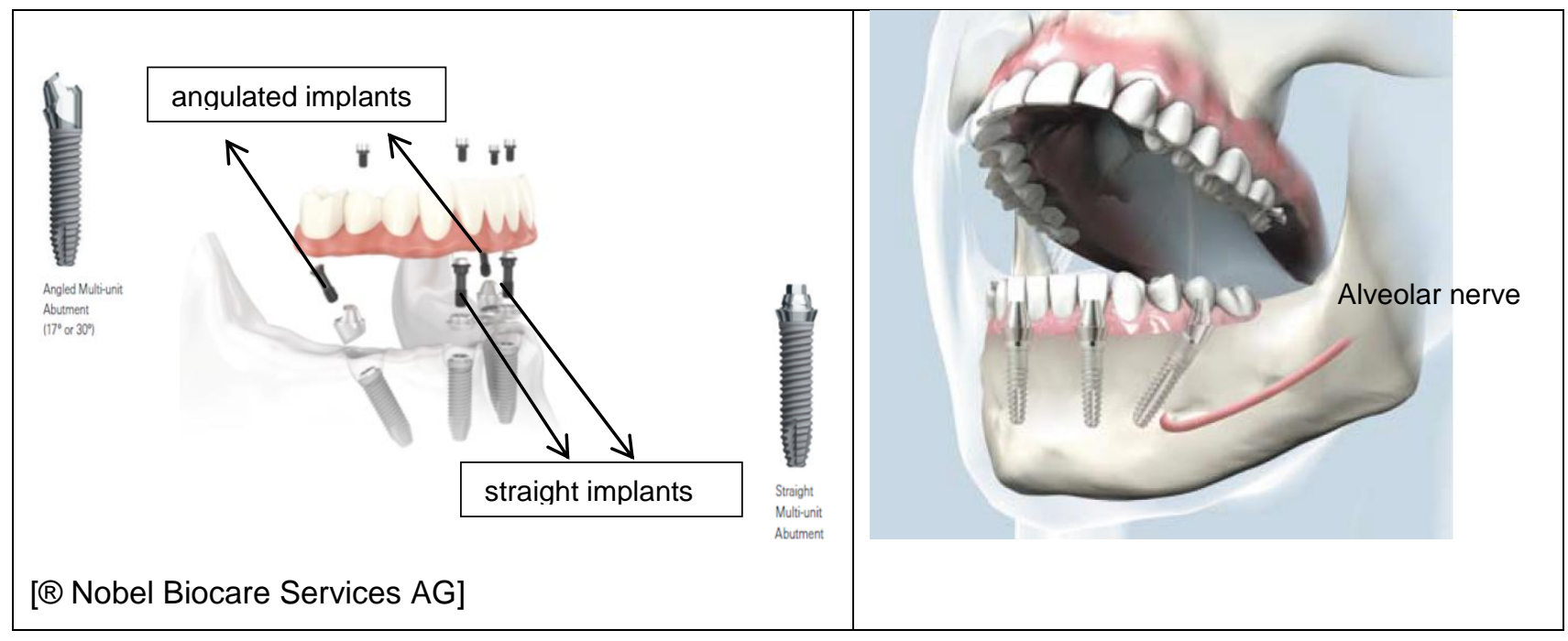

Figure 1. All-on-Four principle [1, 2]

The success of a dental implant depends on the design of the abutment and on the technique by which the abutment screw is placed in the implant. The causes of failure are due to; insufficient bonding between the implant and the surrounding jawbone, failure of the implant tooth fixtures or abutment failure. All depends on the imposed load and the resulting stress: overload can cause bone resorption or fatigue failure of the implant but underloading may lead to bone loss. In cortical bone, stress dissipation is restricted to the immediate area surrounding the implant. In trabecular bone, a broader distant stress distribution occurs. The target compressive stress level for maximum bone growth is at $1.8 \mathrm{MPa}[3,4]$.

To investigate and identify the quality of the implants two methods are used; (i) Simulate the jaw and implant in a finite element model, which is a simplified image of the reality; (ii) experiments in the laboratory where some clinical conditions are simulated but not all of them. When a good outcome has been generated by these methods clinical studies can be carried out.

In an experiment, the mechanical reaction of the implant and the bone are investigated when subjected to loads according the masticatory forces. The reactions worthwhile monitoring are displacements, stresses and strains in the bone, bridge structure and implants. Displacement (and strain) measurements can be performed through Digital Image Correlation. Key to the success of this technology is the realisation of a high quality random speckle pattern onto the studied object. In this paper the preparations for the DIC method are described more in detail. Further investigation on this matter is necessary because an implant is a small specimen and the expected displacements that could lead to failure are very small. Research has shown that micro motion less than 150 to $200 \mu \mathrm{m}$ does not cause failure in osseo-integration [5, 6]. However, most studies have reported that to achieve successful outcomes, the maximum safe motion would be between 100 and $150 \mu \mathrm{m}[7]$.

\section{DIGITAL IMAGE CORRELATION (DIC)}

Digital Image Correlation is an optical method to determine the displacements of an object under load (static as well as dynamic conditions) in two or three dimensions. The method is based on the correlation of two characteristic images of a speckle pattern on the object, where the grey value distribution is analysed. These images are obtained from two high resolution digital cameras.

According to Zhi et al. [8], digital image speckle correlation has the following advantages as compared to other experimental photomechanics techniques such as shadow moiré (SM), projection moiré (PM), moiré interferometry $(\mathrm{MI})$, holographic interferometry $(\mathrm{HI})$, speckle interferometry $(\mathrm{SI})$, electronic speckle pattern interferometry (ESPI), and digital image speckle correlation (DIC).

a) Simple optics based on non-contact and non-destructive measurement technique (compared to $\mathrm{MI}$, $\mathrm{HI}$, and $\mathrm{ESPI})$.

b) Wide range of measurement sensitivity and resolution for macro- and micro-scale deformation measurement (compared to all).

c) Does not require an interferometric laser source and anti-vibration optical table and suitable for both laboratory and field applications (compared to $\mathrm{MI}, \mathrm{HI}, \mathrm{SI}$, and ESPI). 
d) No need for speckle pattern storing, photoelectrical signal conversion, speckle averaging, and signal correlation processing and be able to conduct real-time measurement (compared to SI and ESPI).

e) Computer-aided-measurement technique that can easily be automated (compared to $\mathrm{MI}, \mathrm{HI}, \mathrm{SI}$, and $\mathrm{ESPI})$.

As cited above the expected displacements in dental implant mechanics are very small (magnitude of $100 \mu \mathrm{m}$ ). So DIC on micro-scale is needed. This in combination with the magnitude of the test specimen requires a sufficiently small speckle pattern. The optimal speckle size for accurate DIC measurements is calculated based on the properties of the camera and a rule of thumb, which states that three pixels are needed for one speckle. The resolution of the available camera is 5MPixels (2452 x 2054). The size of the test object is approximately $40 \mathrm{~mm} \times 18 \mathrm{~mm}$.

The optimal speckle size to realise is thus $49 \mu \mathrm{m}$.

$$
\text { optimal speckle size }=\frac{3 \text { pixel }}{\text { speckle }} \operatorname{Max}\left(\frac{l}{2452}, \frac{b}{2054}\right)=48,94 \mu \mathrm{m}
$$

Verplancke's thesis [9] showed that the typical speckle pattern accomplished with paint applied through a spray can isn't sufficiently accurate. Moreover this method depends on circumstances, what means that once a good pattern is obtained it is not reproducible. So other possibilities have to be investigated.

\subsection{Possibilities of Micro-DIC}

In this section a few methods to obtain micro speckles are discussed:

\subsubsection{Polishing and etching}

The realisation of a micro speckle pattern by polishing and etching has been described in literature for titanium, for example by Efstathiou [10]. In this study, first a recrystallization treatment is conducted at 800 ${ }^{\circ} \mathrm{C}$ for $24 \mathrm{~h}$ in a pressurized argon environment. Next, to reveal grain boundaries, the sample is polished using 800 grit SiC paper and etched using e.g. Kroll's reagent (see Table 2. Etchant possibilitiesTable 2). These grains can be used as a pattern for DIC if the microstructure is small enough and if the grain structure can be made sufficiently visible. Using optical microscopy, the obtained grain size was estimated as $30 \mu \mathrm{m}$.

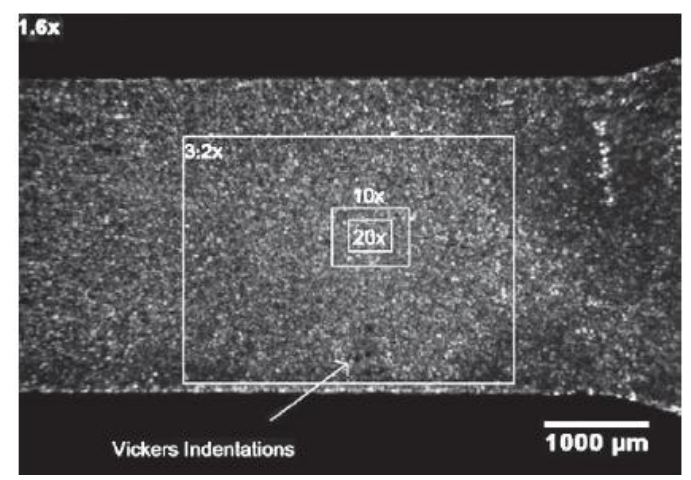

Figure 2. The obtained speckle pattern by Efstathiou [10]

Significant differences in obtained grain structure have been noticed depending on the quality and composition of the titanium (whether it is an alloy, cast titanium or wrought titanium). This is illustrated in Figure 3. 


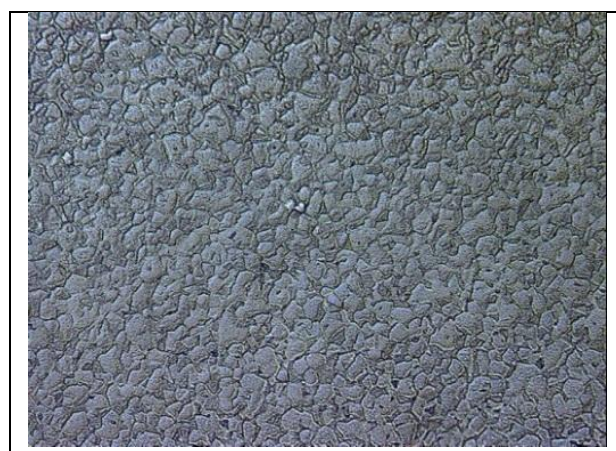

Ti6Al4V titanium alloy, 400X, etched with Kroll's Reagent

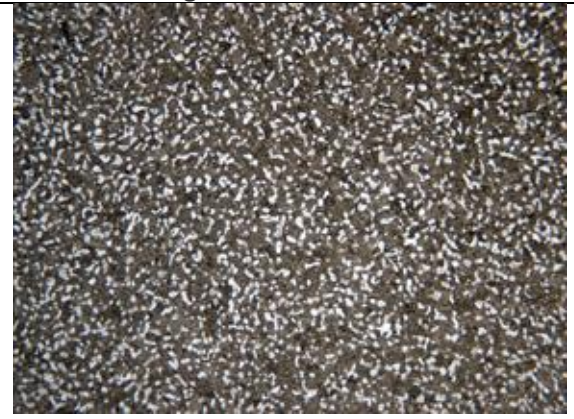

Wrought Titanium, 200X, Etchant Ammonium Bifluoride.

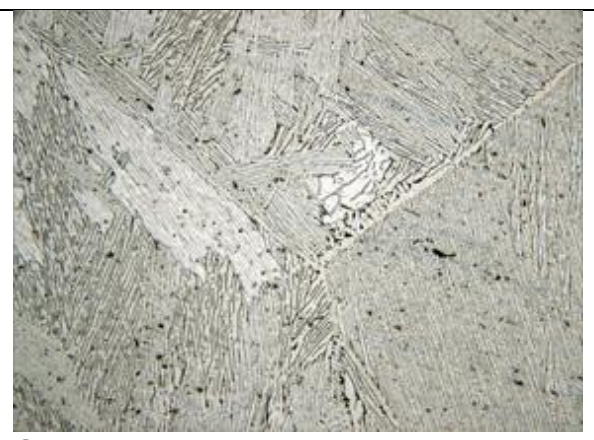

Cast Titanium, 200X, Etched with Kroll's Reagent.

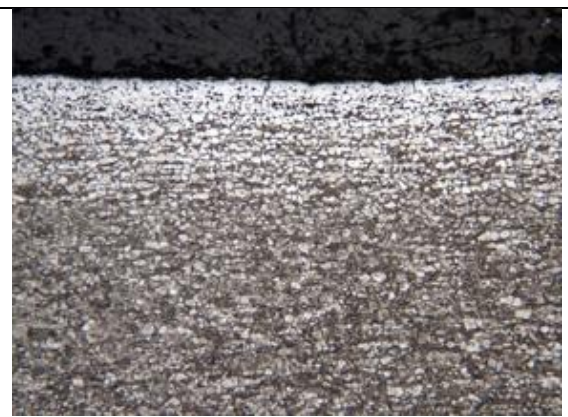

Alpha-rich Case Wrought Titanium, 200X without echant.

Figure 3. Microstructures of Titanium after polishing and etching [11]

These pictures clearly show that for every different quality or type of titanium other preparation processes are needed. The most common polishing and etching methods for titanium are listed in the tables below (Table 1 and Table 2). Polishing can be done manually or automatic, the methods described in Table 1 are mostly for automatized polishing. Regarding the etching, caution is needed because titanium is etched with strong and therefore dangerous acids. A flue and adequate protection must be provided. Chemical etching selectively attacks specific microstructural features, like inclusions. Common etching techniques are swabbing (which is less aggressive and submits a controlled attack) and immersion (submerging the specimen this produces a more uniform etching but it is easier to under of over etch, so good timing is required).

Table 1. Polishing possibilities [11]

\begin{tabular}{|l|l|l|l|l|}
\hline Abrasive/surface & Lubricant & Force $[\mathbf{k g}]$ & $\begin{array}{l}\text { Speed } \\
\text { [rpm] }\end{array}$ & Time \\
\hline $\begin{array}{l}\mathbf{2} \mathbf{6 0} \text { grit SiC paper } \\
\mathbf{3 6 0} \text { grit SiC paper } \\
\mathbf{6 0 0} \text { grit SiC paper } \\
\mathbf{8 0 0} \text { grit SiC paper } \\
\mathbf{1 2 0 0} \text { grit SiC paper }\end{array}$ & Water & $2.27-4.54$ & 200 & $\begin{array}{l}\text { Until plane } \\
1 \text { minute } \\
1 \text { minute } \\
1 \text { minute } \\
1 \text { minute }\end{array}$ \\
\hline $\begin{array}{l}\mathbf{6} \boldsymbol{\mu m} \text { DIAMAT } \\
\text { diamond on } \\
\text { TEXPAN polishing } \\
\text { pad }\end{array}$ & $\begin{array}{l}\text { DIALUBE } \\
\text { Purple } \\
\text { Extender }\end{array}$ & $2.27-4.54$ & 200 & 3 minutes \\
$\begin{array}{l}\mathbf{\mu m} \text { DIAMAT } \\
\text { GOLDPAD } \\
\text { polishing pad }\end{array}$ & $\begin{array}{l}\text { DIALUBE } \\
\text { Purple }\end{array}$ & $2.27-4.54$ & 200 & 2 minutes \\
\hline $\begin{array}{l}\mathbf{0 . 0 5} \text { um Nanometer } \\
\text { alumina on } \\
\text { ATLANTIS polishing } \\
\text { pad }\end{array}$ & & $2.27-4.54$ & 100 & 1 minute \\
\hline
\end{tabular}


Table 2. Etchant possibilities [11]

\begin{tabular}{|c|c|c|c|c|}
\hline Etchant & Composition & Conc. & Conditions & Comments \\
\hline Kellers Etch & $\begin{array}{l}\text { Distilled water } \\
\text { Nitric acid } \\
\text { Hydrochloric acid } \\
\text { Hydrofluoric acid }\end{array}$ & $\begin{array}{l}190 \mathrm{ml} \\
5 \mathrm{ml} \\
3 \mathrm{ml} \\
2 \mathrm{ml}\end{array}$ & $\begin{array}{l}10-30 \text { second } \\
\text { immersion }\end{array}$ & $\begin{array}{l}\text { Excellent for aluminum } \\
\text { and titanium alloys }\end{array}$ \\
\hline $\begin{array}{l}\text { Kroll's } \\
\text { Reagent }\end{array}$ & $\begin{array}{l}\text { Distilled water } \\
\text { Nitric acid } \\
\text { Hydrofluoric acid }\end{array}$ & $\begin{array}{l}92 \mathrm{ml} \\
6 \mathrm{ml} \\
2 \mathrm{ml}\end{array}$ & $\begin{array}{l}\text { Swab specimen } \\
\text { up to } 20 \text { seconds }\end{array}$ & $\begin{array}{l}\text { Excellent for titanium } \\
\text { and alloys }\end{array}$ \\
\hline $\begin{array}{l}\text { Modified } \\
\text { Weck's } \\
\text { reagent }\end{array}$ & $\begin{array}{l}\text { Distilled water } \\
\text { Ethanol } \\
\text { Ammonium bifluoride }\left(\mathrm{NH}_{4} \mathrm{HF}_{2}\right)\end{array}$ & $\begin{array}{l}100 \mathrm{ml} \\
50 \mathrm{ml} \\
2 \mathrm{~g}\end{array}$ & Swab 15-20s & $\begin{array}{l}\text { Modified for titanium. } \\
\text { Gives more colour and } \\
\text { contrast }\end{array}$ \\
\hline
\end{tabular}

\subsubsection{Silicon}

A speckle pattern is achieved by dispersing silicon microparticles (Si) in the air and collecting them into a die with the samples in it [12].

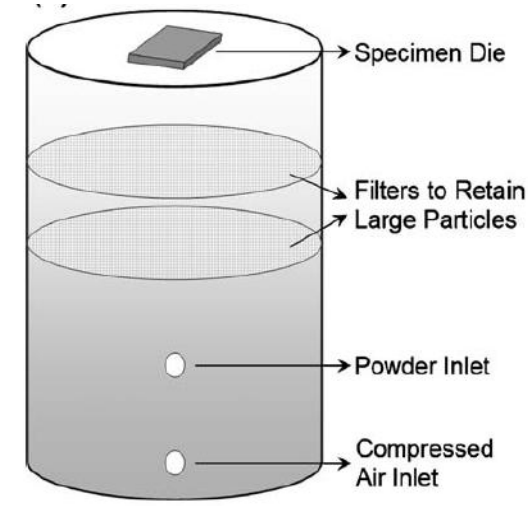

Figure 4. How to apply the silicon microparticles [12]

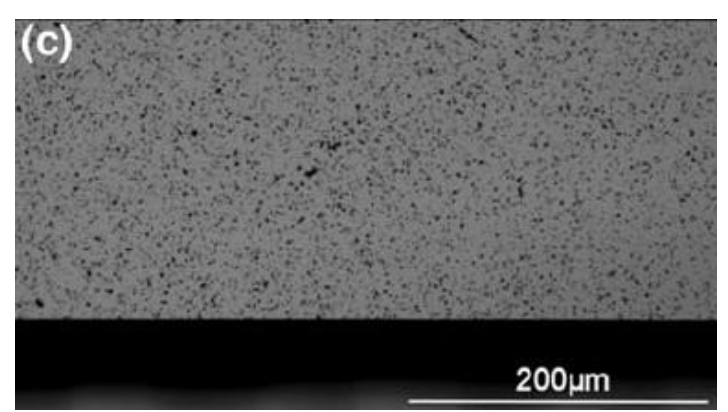

Figure 5. The obtained speckle pattern [12]

Figure 4 shows a hollow cylindrical shaft with side inlets to introduce compressed air at the bottom of the cylinder and sub-microscale particles above the air inlet. The average particle size in the dispersed silicon powder was $1 \mu \mathrm{m}$, but due to hydrophobic interactions the particles formed large agglomerates. The use of filters eliminated large agglomerates. The dies with Platinum specimens were placed at the top of the cylinder to collect the particles forced by the compressed air through the topmost filter. The micro-particles adhere onto the freestanding surfaces by van der Waals forces and static charges.

A variant of silicon, namely silicon dioxide (or silica, $\mathrm{SiO}_{2}$ ) can also be used. $\mathrm{SiO}_{2}$ is a nano-composite with a particle size of $62.5 \mu \mathrm{m}$ average. This is applied through a film containing the $\mathrm{SiO}_{2}$ particles, often on polymers. The danger to apply a film on metal materials is reflection; high reflection makes correlation of the images through DIC very hard and impossible to find accurate results.

\subsection{3 $\mathrm{TiO}_{2}$}

$\mathrm{TiO}_{2}$ has been used to generate speckle patterns on teeth and teeth crowns made of composites. In [13] $\mathrm{TiO}_{2}$ powder of particle size less than $10 \mu \mathrm{m}$ is sprinkled on the tooth surface to generate a pattern. Deformations of $20 \mu \mathrm{m}$ haven been measured with the $\mathrm{DIC}$ method. $\mathrm{TiO}_{2}$ is especially useful because it also reduces light reflection under ambient light. The application of $\mathrm{TiO}_{2}$ on the surface is done through a spray powdering aid.

In the same article nano-copper particles and $100 \mu \mathrm{m}$ sized carbon particles are rejected because of the inappropriate size.

\subsubsection{Focused ion beam (FIB)}

FIB is a technique for site-specific analysis, deposition, and ablation of materials. The importance of FIB in the area of DIC is the ability to apply markers of typical dimensions from $20 \mathrm{~nm}$ to several micrometres on materials (both ion and electron beam assisted deposition of metals e.g. Pt, W, Fe, Co, Au). These markers can achieve a random pattern usable for digital image correlation $[14,15]$. 
In the in vitro test a titanium bridge structure will be used. So the micro-DIC speckle pattern should be applied on titanium. The application and mainly the sticking of powder on the surface of the titanium bridge are challenging. Realisation of a random pattern on all the surfaces seems impossible. So the powder concepts discussed in 2.1.2 and 2.1.3 are not further exploded. Etching and polishing can be a good solution if the microstructure of titanium is small enough and the preparation processes are feasible. Therefore the etching and polishing methods are first tried out on a test bridge (Figure 7) to see if the obtained speckle pattern meets the requirements of the available DIC equipment.

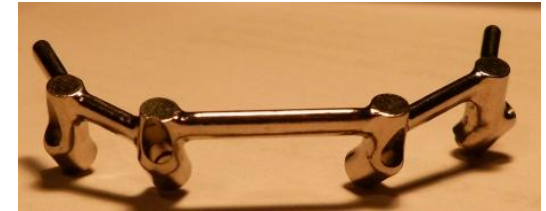

Figure 6. Bridge structure $(40 \mathrm{~mm} \times 10 \mathrm{~mm})$

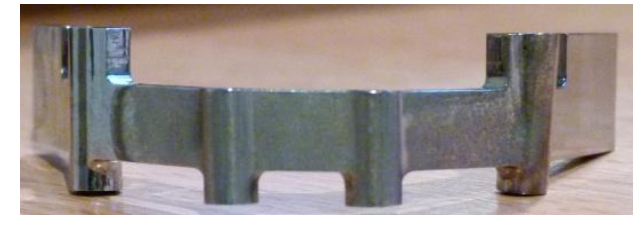

Figure 7. Test bridge structure $(40 \mathrm{~mm} \times 18 \mathrm{~mm})$

The test bridge is already polished and can be etched immediately. In order to find the best pattern a few combinations of etchants are tested out. As calculated above the optimal speckle size is $49 \mu \mathrm{m}$, this should be the average grain size. This grain size appears not to be a real problem but achieving an appropriate contrast is. Normally when DIC is used, the specimen is first painted white and after that a black speckle pattern is made. Realisation of this kind of contrast with the microstructure of titanium appears impossible. Through varying the etching time and etchant, differences in contrast can be realised. It is seen that a combination of reagents provides the best contrast, namely Kroll's reagent to visualize the grain boundaries and modified Weck's reagent to colour the microstructure. The best obtained results are shown in Table 3. So the specimen has to be polished and the best combination of the reagents has to be applied on the bridge. Polishing a 3D structure of this shape is not possible with a machine, so electrolytic polishing will be tried out. The pictures have been imported in the DIC software and were recognized and accepted. Whether the program is able to correlate will be tested in the following period.

Table 3. Etching results

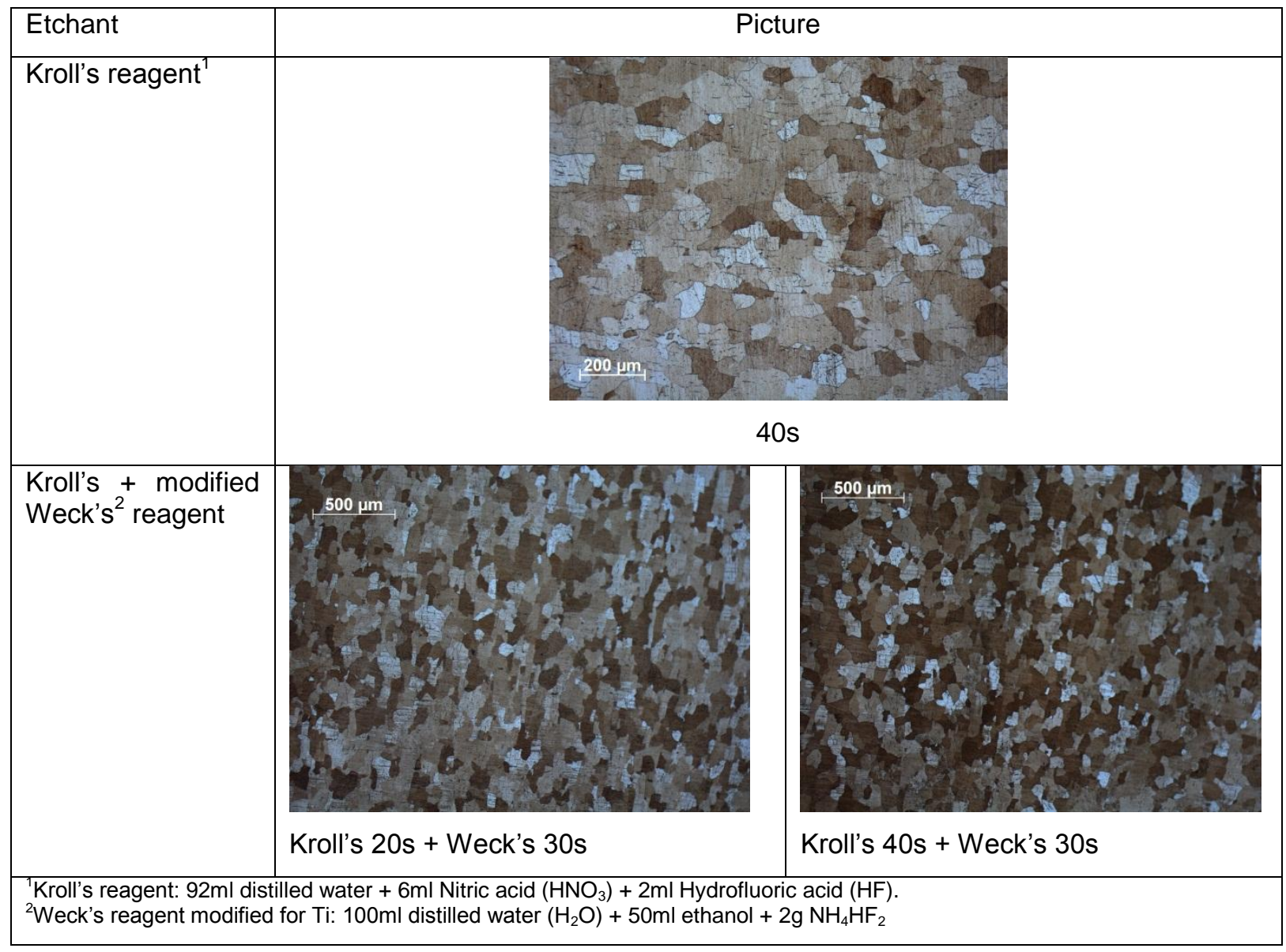


Based on the evaluation of micro speckle patterns and accomplished patterns, further tests will be carried out. First the test specimen will be polished again and the full bridge will be etched with the optimal procedure (a combination of Weck's and Kroll's etchants ). Then Digital Image Correlation will be carried out to see if the obtained contrast is sufficient under the light conditions in the lab or if polarized light is needed.

When that is accomplished the experiments can start. The results hereof will be compared to a result obtained from a finite element analysis simulating the experimental setup and conditions.

\section{ACKNOWLEDGEMENTS}

The authors would like to acknowledge the support Michel De Waele for his help with the polishing and etching.

\section{REFERENCES}

1. Nobel Biocare Services AG, All-on-4, TREATMENT CONCEPT FOR EDENTULOUS ARCHES, N.B.S. AG, Editor 2010.

2. Bredent medical, SKY fast \& fixed, in SKY IMPLANT SYSTEM, B. medical, Editor: Senden, Germany.

3. Geng, J.P., K.B. Tan, and G.R. Liu, Application of finite element analysis in implant dentistry: a review of the literature. J Prosthet Dent, 2001. 85(6): p. 585-98.

4. Kalanovic, M. and N. Zdravkovic-Petrovic, Three-dimensional finite element stress analysis of SKY implant system. Journal of the Serbian Society for Computational Mechanics, 2010. 4(2): p. 87-96.

5. Pilliar, R.M., J.M. Lee, and C. Maniatopoulos, Observations on the Effect of Movement on Bone Ingrowth into Porous-Surfaced Implants. Clinical Orthopaedics and Related Research, 1986(208): p. 108-113.

6. Viceconti, M., et al., Large-sliding contact elements accurately predict levels of bone-implant micromotion relevant to osseointegration. Journal of Biomechanics, 2000. 33(12): p. 1611-1618.

7. Fazel, A., et al., Micromotion and Stress Distribution of Immediate Loaded Implants: A Finite Element Analysis. Clinical Implant Dentistry and Related Research, 2009. 11(4): p. 267-271.

8. Shi, X.Q., et al., In-situ micro-digital image speckle correlation technique for characterization of materials' properties and verification of numerical models. leee Transactions on Components and Packaging Technologies, 2004. 27(4): p. 659-667.

9. Verplancke, K., Testing protocol for in vitro evaluation of dental implants, in Department of Mechanical Construction and Production 2011, University Ghent: Ghent. p. 121.

10. Efstathiou, C., H. Sehitoglu, and J. Lambros, Multiscale strain measurements of plastically deforming polycrystalline titanium: Role of deformation heterogeneities. International Journal of Plasticity, 2010. 26(1): p. 93-106.

11. PACE Technologies. Metallographic. Available from: http://www.metallographic.com.

12. Jonnalagadda, K.N., et al., Experimental Investigation of Strain Rate Dependence of Nanocrystalline Pt Films. Experimental Mechanics, 2010. 50(1): p. 25-35.

13. Chuang, S.F., T.Y. Chen, and C.H. Chang, Application of digital image correlation method to study dental composite shrinkage. Strain, 2008. 44(3): p. 231-238.

14. Winiarski, B. and P.J. Withers, Micron-Scale Residual Stress Measurement by Micro-Hole Drilling and Digital Image Correlation. Experimental Mechanics, 2011.

15. van Kouwen, L., A. Botman, and C.W. Hagen, Focused Electron-Beam-Induced Deposition of $3 \mathrm{~nm}$ Dots in a Scanning Electron Microscope. Nano Letters, 2009. 9(5): p. 2149-2152. 